study has suggested that PT may be able to make a useful contribution to improving public health policy evaluations.

\section{OP114 ASSOCIATION OF DRUG PRESCRIPTIONS WITH INCIDENT DEMENTIA}

${ }^{1} \mathrm{C}$ Schnier*, ${ }^{1,2} \mathrm{~T}$ Wilkinson, ${ }^{1} \mathrm{~K}$ Bush, ${ }^{1} \mathrm{~A}$ Hutchinson, ${ }^{1} \mathrm{~K}$ Rannikmäe, ${ }^{1,2} \mathrm{CLM}$ Sudlow. ${ }^{1}$ Usher Institute of Population Health Sciences and Informatics, University of Edinburgh, Edinburgh, UK; ${ }^{2}$ Centre for Clinical Brain Sciences, University of Edinburgh, Edinburgh, UK

\subsection{6/jech-2019-SSMabstracts.113}

Background Recent studies have reported conflicting associations between drug prescriptions and incident dementia. Any association between drug and dementia could be due to the drugs directly causing or preventing dementia; the drugs being associated with a risk factor for dementia; or the drugs being prescribed as a consequence of prodromal dementia. Based on methodology developed for genome-wide association studies, we systematically analyzed the effect of 733 drugs on incident dementia in a population-wide linkage study and clinically reviewed the associations.

Methods Using linked, routinely-collected electronic health records from hospital admissions, mortality records and primary care consultations, we followed-up 574,237 Welsh residents from their 60th birthday onwards to classify exposure (drug prescriptions) and dementia incidence. During follow-up, $13,786(2.4 \%)$ of the study population developed dementia. We used time-dependent Cox proportional hazard models to study the effect of exposure on dementia incidence, controlling for the effects of age, sex, year, deprivation and smoking status. To account for multiple testing, we first analyzed a $50 \%$ household-area stratified random sample of the study population (discovery cohort), selected results with a Bonferroni-corrected $p$-value, re-run the analysis of 'significantly' associated drugs in the remaining 50\% (validation cohort) and once again selected results with a Bonferroni-corrected $p$-value. We displayed the results (hazard ratio and $p$-value) from the complete cohort in several stratified volcano-plots and clinically reviewed the findings to identify potential pathways of effect.

Results $177 / 733$ (24\%) of the analysed drugs were significantly associated with dementia incidence. Of those, 7 were for neurodegenerative conditions that can cause dementia, 14 were for vascular diseases, 13 for diabetes, 16 for depression and 39 for symptoms or complications of dementia. Only four, all travel-related vaccines, were associated with a lower dementia incidence. Some drugs associated with an increased hazard of dementia clustered around several unexpected indications, including: gastro-oesophageal reflux disease, altered bowel habit, lower urinary tract symptoms and infections, anxiety, sleep disturbance, pain and nausea/vertigo.

Discussion By grouping drugs by indication, we identified several drugs with a potential of having a direct association with increased risk of dementia. We also identified drugs which are related to (known) risk factors for dementia, including those prescribed for cardiovascular disease and diabetes. The effect of travel-related vaccines is puzzling and might be more related to a preventative association of travelling with dementia incidence. Most interestingly, we identified several drugs which might have been prescribed as a consequence of a preclinical, non-cognitive syndrome in dementia.

\section{OP115 IMPROVING THE ASSESSMENT OF CAUSALITY IN POPULATION HEALTH: SHOULD BRADFORD HILL BE REVISITED TO INCORPORATE DEVELOPMENTS IN CAUSAL INFERENCE?}

M Shimonovich*, H Thomson, A Pearce, V Katikireddi. MRC/CSO Social and Public Health Sciences Unit, University of Glasgow, Glasgow, UK

\subsection{6/jech-2019-SSMabstracts.114}

Background Bradford Hill's (BH) guidelines are the traditional approach to causal assessment in population health and epidemiology. However, assessments can be inconclusive; there is no consensus on the thresholds required for components. Some have proposed incorporating more recent developments in causal thinking to $\mathrm{BH}$ guidelines to improve assessment of causality. This study aims to understand how traditional approaches to causal assessment can be refined by incorporating alternative causal methods. We will do this by understanding the similarities and differences of these approaches to $\mathrm{BH}$. Methods We mapped each $\mathrm{BH}$ component against three subsequent, prominent causal inference approaches: directed acyclic graphs (DAGs), grading of recommendations, assessment, development and evaluation methodology (GRADE), and sufficient-component cause models (SCC, also referred to as 'causal pies'), drawing upon existing studies that had assessed the overlap between one or more of these approaches. Existing studies were found through targeted searching and snowballing, with no a priori list of inclusion/exclusion criteria.

Results The approaches can be grouped into two categories: models (DAGs and SCC) and assessment guidelines (BH and GRADE). The literature does not necessarily explicitly make this distinction, but the identified literature largely restricted comparisons within each of these categories.

We found that some components overlap between the guidelines and models, while some are specific to certain approaches. For example, BH causal assessment considers if an increased exposure corresponds with increased incidence of the disease (dose-response). Similarly, GRADE will upgrade evidence from an observational study with evidence of doseresponse. However, testing dose-response for DAGs may not be helpful. A dose-response may be demonstrated for different exposure levels due to a confounder that has the same impact on the exposure and the outcome. Thus, it would be the confounder causing the dose-response, not the causal relationship. The SCC model is often drawn with binary exposures and outcomes where dose-response is not considered. However, it can be incorporated by including dose as providing different contributions to the causal pie. Similar comparisons were made for the remaining $\mathrm{BH}$ components.

Conclusion Assessing causal relationships is challenging, yet of fundamental importance. There have been limited efforts to incorporate insights from DAGs and SCC into BH guidelines. However, our review did not investigate all potential approaches to assessing causality (e.g. International Agency for Research on Cancer) and the comparisons require further analysis. Nevertheless, this detailed exploration improves the 